Hough Transform Based Corner Detection for Laser Beam

Positioning

A. A. S. Awwal

July 29, 2005

SPIE - Optics and Photonics 2005

San Diego, CA, United States

July 31, 2005 through August 4, 2005 
This document was prepared as an account of work sponsored by an agency of the United States Government. Neither the United States Government nor the University of California nor any of their employees, makes any warranty, express or implied, or assumes any legal liability or responsibility for the accuracy, completeness, or usefulness of any information, apparatus, product, or process disclosed, or represents that its use would not infringe privately owned rights. Reference herein to any specific commercial product, process, or service by trade name, trademark, manufacturer, or otherwise, does not necessarily constitute or imply its endorsement, recommendation, or favoring by the United States Government or the University of California. The views and opinions of authors expressed herein do not necessarily state or reflect those of the United States Government or the University of California, and shall not be used for advertising or product endorsement purposes. 


\title{
Hough transform based corner detection for laser beam positioning
}

\author{
Abdul A. S. Awwal \\ National Ignition Facility \\ University of California, Lawrence Livermore National Laboratory, Livermore, CA. 94551 \\ E-mail: awwal1@1lnl.gov
}

\begin{abstract}
In laser beam alignment in addition to detecting position, one must also determine the rotation of the beam. This is essential when a commissioning new laser beam for National Ignition Facility located at the Lawrence Livermore National Laboratory. When the beam is square, the positions of the corners with respect to one another provides an estimate of the rotation of the beam. This work demonstrates corner detection in the presence or absence of a second order non-uniform illumination caused by a spatial mask. The Hough transform coupled with illumination dependent pre-processing is used to determine the corner points. We show examples from simulated and real NIF images.
\end{abstract}

Key word: Corner detection, line detection, Hough transform, Hough filtering, automated optical alignment, beam rotation, image processing, image quality detection processing

\section{INTRODUCTION}

The National Ignition Facility (NIF), currently under construction at the Lawrence Livermore National Laboratory, is a stadium-sized facility containing a 192-beam, 1.8-megajoule, 500-terawatt, ultraviolet laser system for the study of inertial confinement fusion and the physics of matter at extreme energy densities and pressures [1]. The alignment of this high energy pulsed laser is a critical process requiring accurate measurement. An automatic alignment (AA) system is designed and implemented to ensure successful delivery of high energy pulse in each of the 192 beams. During the commissioning of new beam lines, the amount of beam rotation needs to be monitored and corrected. One such image appears in the pre-amplifier module (PAM) as shown in Figure 1. In this beam the fiducials corresponding to the reference and beams are located outside the area of the main beam. In this paper, we describe the algorithm for determining the beam rotation from the locations of the four corners of the central beam.

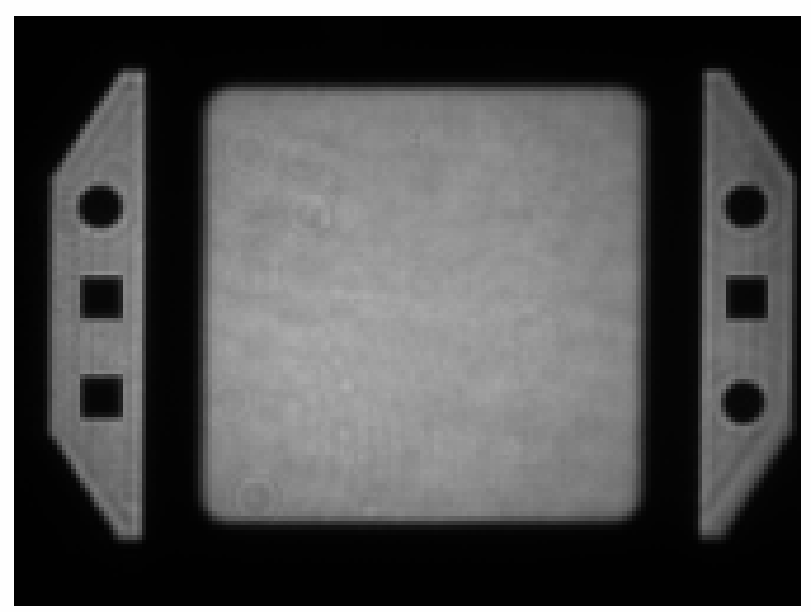

Fig. 1: The PAM output beam showing six fiducials and the square main beam. 


\section{BACKGROUND}

Hough transform (HT) is a technique for detecting features of a particular shape within an image [2,3]. In one application of HT, it allows detection lines in an image. In practical implementation of Hough transform [3] each line is represented by two parameters, namely $r-\theta$, which are the distance and the angle of the normal to the to-be-detected line from the center of the co-ordinate system as shown in Fig. 2. With these parameters the equation of the straight line

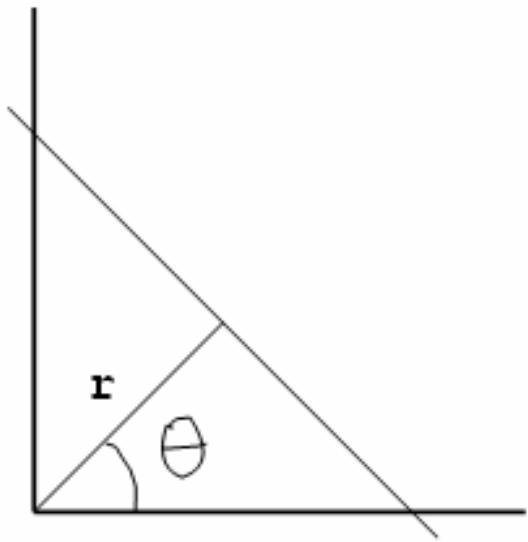

Fig. 2: A typical line showing the parameters for Hough transform. 
is given by

$$
x \cos \theta+y \cos \theta=d
$$

Note that a line can be represented by a single $r-\theta$ value. A point is treated as intersections of infinite number of lines. By calculating the value of $r$ for every possible $\theta$ value for all these lines through a point, a sinusoid curve is created in the $r-\theta$ plane, also known as the Hough plane. The contributions from all such points are accumulated in the Hough space. Two points lying on the same line must have a common $r$ and $\theta$ values. Thus the two sinusoids corresponding to these points belonging to a line intersect at a common $\left(r_{i}, \theta_{i}\right)$ in the parameter space. Thus any straight line with a fixed $\left(r_{i}, \theta_{i}\right)$ value will appear to have a high peak in the accumulated Hough space, which will be proportional to the number of pixels belonging to the line. Detecting the peak in the Hough space, detects the straight lines with the corresponding $\left(r_{i}, \theta_{i}\right)$ value. When lines representing edges of objects are to be found, usually an edge detector is applied to reduce the amount of information and the background noise. Note that matched filtering based technique will not be appropriate [4] here since the characteristics of each of the corners are different. It may need a large number of templates to encompass all the variations.

\section{ALGORITHM FOR A UNIFORMLY ILLUMINATED BEAM}

A typical example of square beam used in the preamplifier module with internal and external fiducials is shown in Figure 1 . The beam is fairly uniform, without showing any significant variation of image intensity. An initial smoothing followed by a sobel edge operator and a subsequent thresholding produces the image shown in Fig. 2.

The four edges of the main beam does not exhibit any significant deviation from straight line. Performing a Hough transform of the edge image produces the image shown in Fig. 3. Using the reflection property of Hough transform (HT), the right most side of the HT is folded onto the left side.

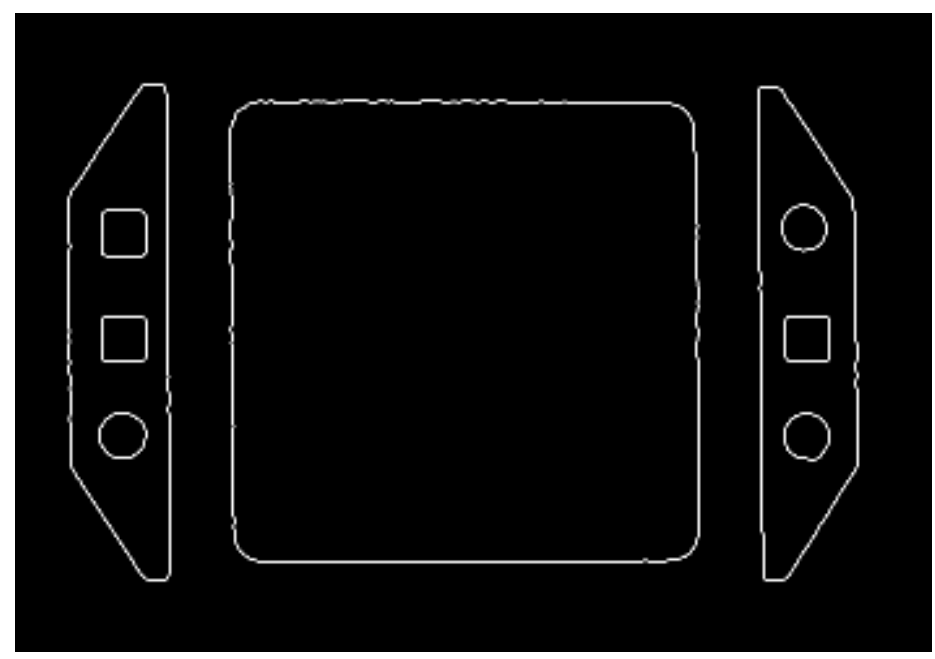

Fig. 3: The edge image detected from image of figure 1

The Hough space shows clearly the presence of six peaks corresponding to vertical lines at $\theta$ of zero degree. The horizontal lines correspond to 90 (1.57 radians) degrees show two distinct peaks. The locations of these peaks are selected after applying a polynomial interpolation through the peak values. In order to reduce the ambiguity, the expected locations of the lines are initially filtered using a band pass filter in the Hough domain. The angular $(\theta)$ 
bandwidth of the filter determines the maximum angular deviation of the straight lines from a pure vertical or horizontal orientation that can be detected. In the algorithm, the center wavelength and the bandwidth of the filter is a changeable parameter, which can be modified to detect a line deviated from perfect horizontal or vertical orientation.

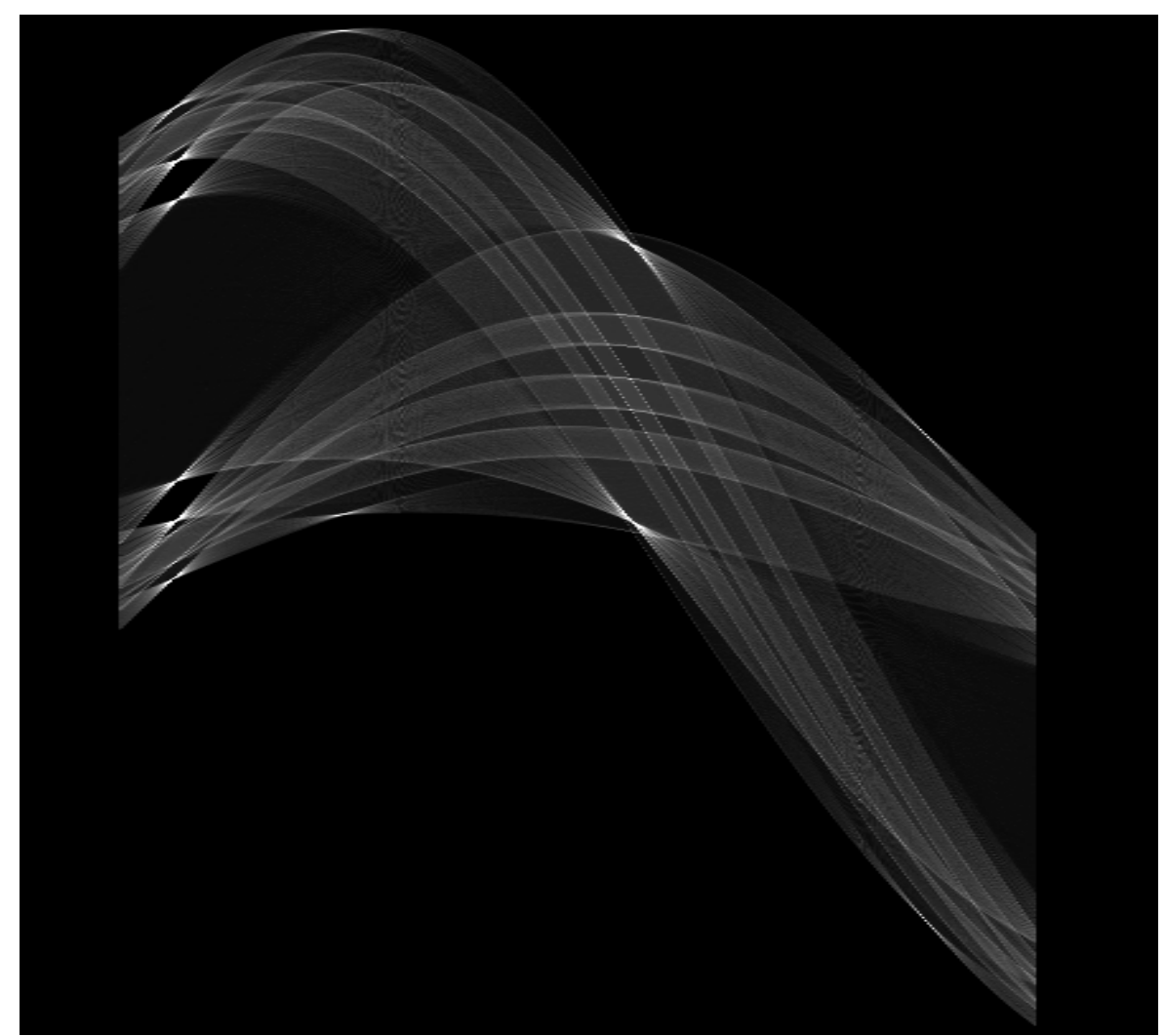

Fig. 4: The Hough transform of the edge image shown in figure 2. (image enhanced for better viewing)

As shown in the Fig. 4 and Fig. 5, there are six vertical lines. Four of these lines are from the edges of the side wings of the beam. The desired lines are those that correspond to the main square beam. The correct lines are chosen from the Hough space using a criterion of line spacing between two consecutive lines. This line spacing test filters out the unwanted vertical lines. Thus the algorithm selects inner two bright spots with the proper spacing. The algorithm has a spacing threshold in addition to the expected spacing parameter. If the beam magnification changes and causes the two lines to be wider apart then the algorithm will not be able to identify the two lines. This is desirable since it may flag an unexpected change in the magnification or hardware, which may need to be investigated. 


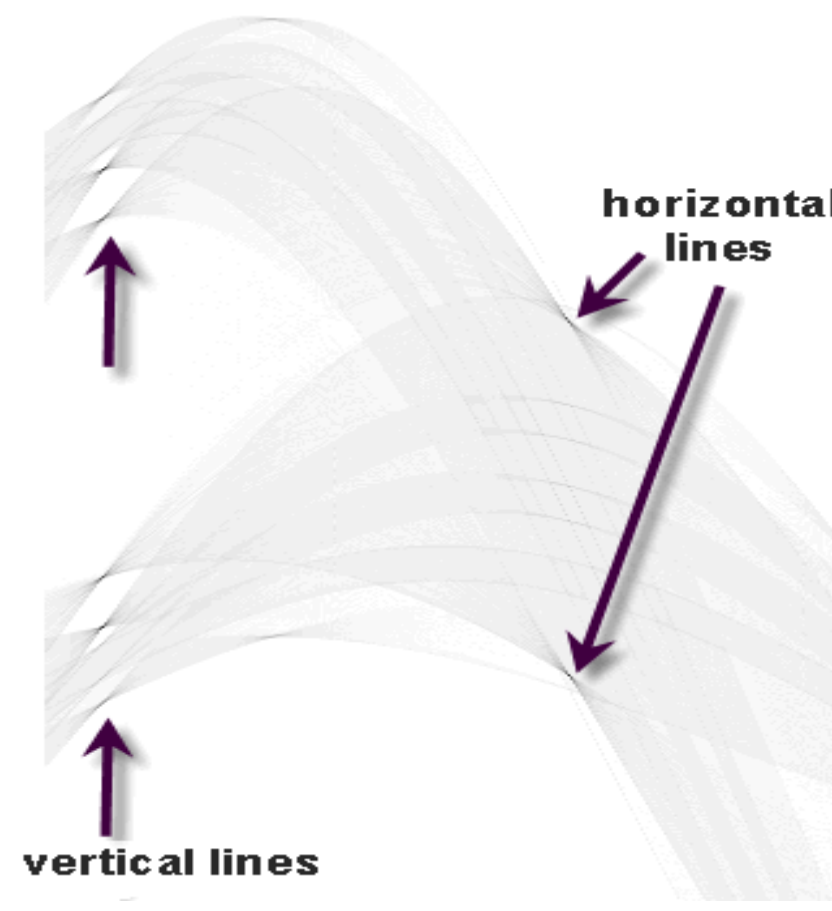

Fig. 5: The Hough transform of the edge image shown in figure 2 inverted.

\section{ALGORITHM FOR A BEAM WITH SECOND ORDER ILLUMINATION GRADIENT}

When an attenuator mask is placed on the beam-path, it causes a change in the beam profile as shown in Figs. 6 and 7 . From Fig. 6, the beam shows darker region inside the beam and lighter near the edge. The average cross-section depicts a parabolic intensity distribution in the horizontal direction. As a result, the darker region near the center approaches values close to those of the background region near the edges. Consequently, when the beam is thresholded, the horizontal edges become curved instead of a being straight as shown in Fig. 8. In the Hough space, no distinct lines are detected exhibiting a strong peak, instead small line segments oriented in several different directions cause the peaks to spread over a range. As such the algorithm fails to pick up any horizontal lines corresponding to highest peak.

It was found after some experimentation that a logarithmic filtering can enhance the edge straightness by enhancing the contrast near the low intensity level. However, the same filtering for a uniform beam will result in losing the vertical edges. In order to overcome this problem, we devised a way to estimate the beam nonuniformity. A horizontal line

segment is generated $\bar{I}_{y}(x):=\sum_{j=1}^{N_{y}} I\left(x_{j}, y_{k}\right) ; j=1, \cdots, N_{x}$ for $\quad \bar{I}_{y} \in R^{N_{x} \times 1}$

by column averaging the beam. The variation of the intensity along this segment measured by the variance is estimated as an estimate of the nonuniformity of the beam. When the beam is nonuniform only then we apply the logarithmic image enhancement.

In the absence of any nonlinear processing on the image, the binarization of the above image shows that the top and the bottom edge is curved inwards as depicted in Fig. 8. A Hough transform of the edge image fails to determine the horizontal lines, because there is none. 


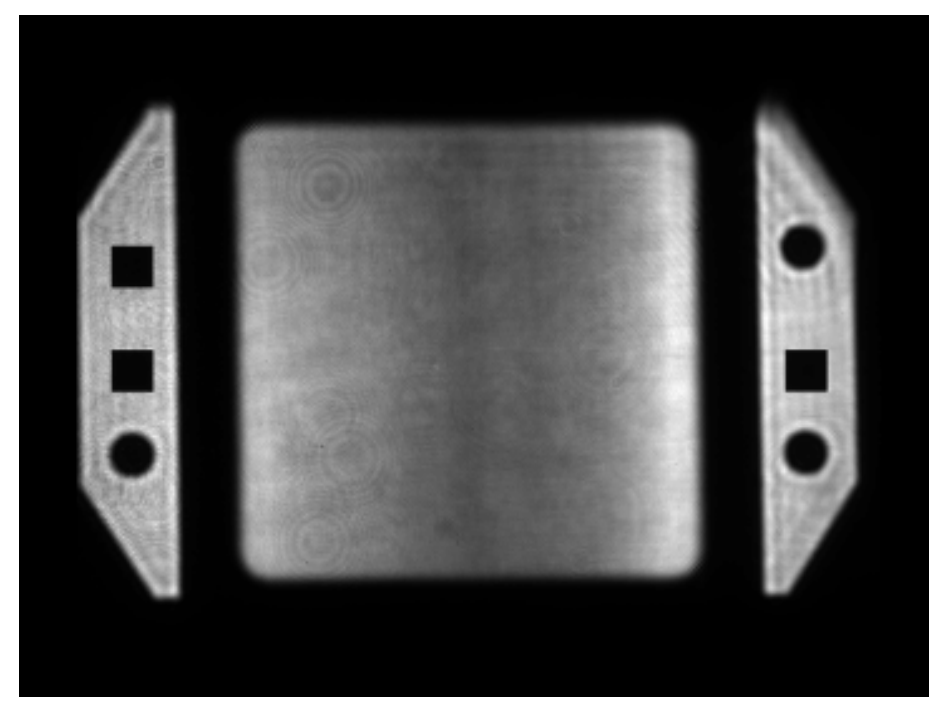

Fig. 6: The PAM image with second order intensity gradient

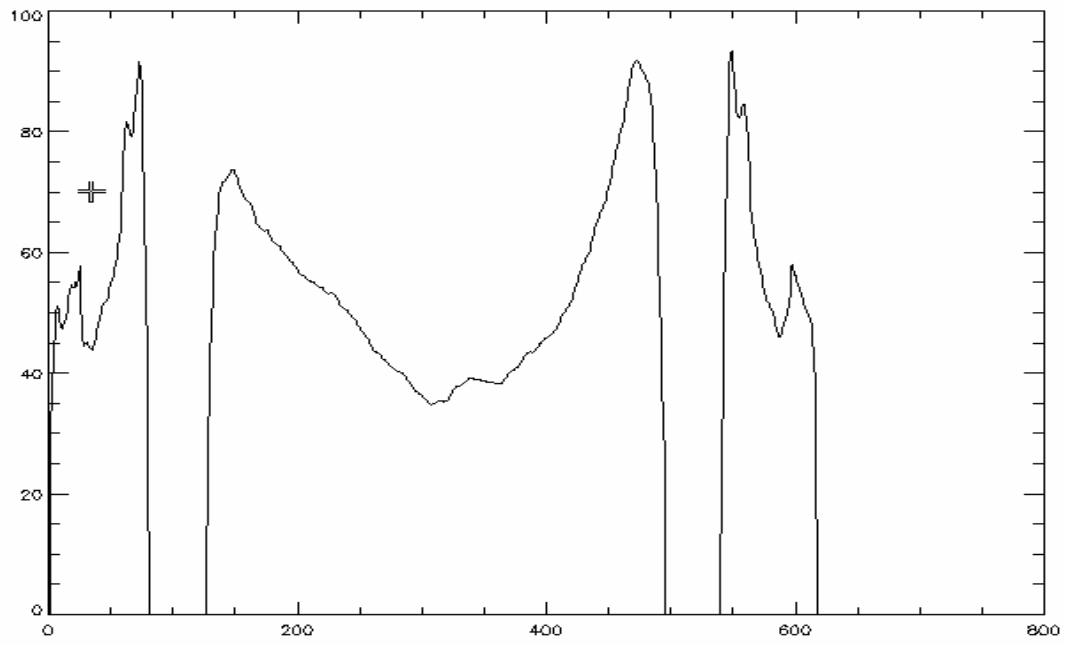

Fig. 7: The average cross-section of PAM image showing second order intensity gradient

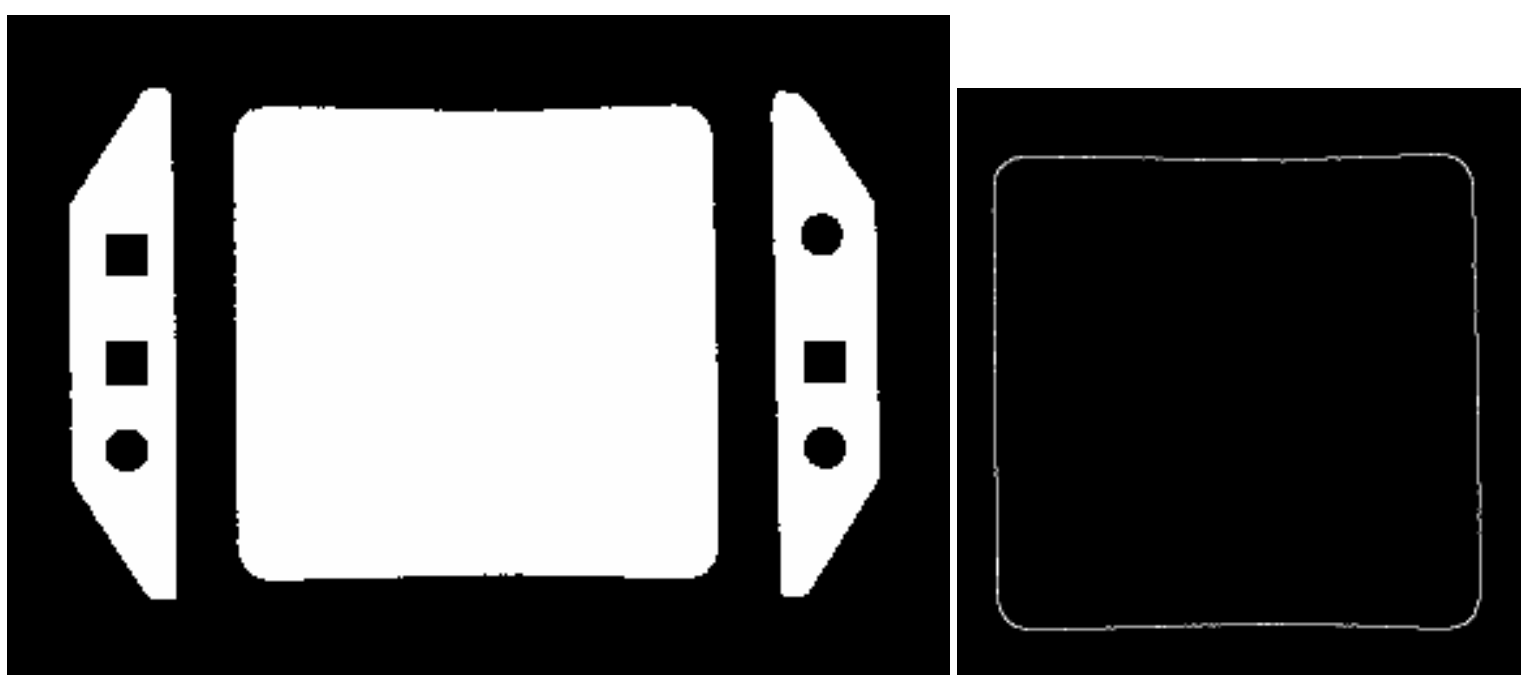

Fig. 8: The binarized PAM image with corresponding edge image 


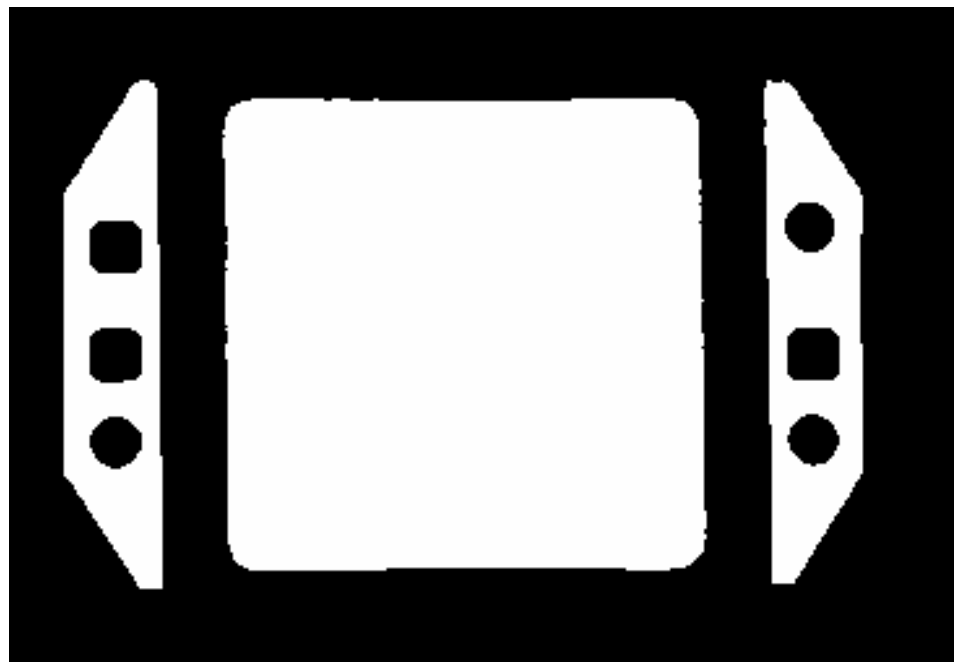

Fig. 9: The binarized PAM image after preprocessing with log filter

As mentioned earlier, a logarithmic processing after evaluating the image quality allows us to enhance the low contrast region. As a result, thresholding produces a horizontal edge as shown in Fig. 9. The corresponding Hough transform shows presence of distinct peaks as shown in Fig. 10. Note that the peaks appear at the center of the pass band in the Hough space which are centered around 0 and 90 degrees. The numerical values of these peaks as generated by the algorithm is shown in Fig. 11.

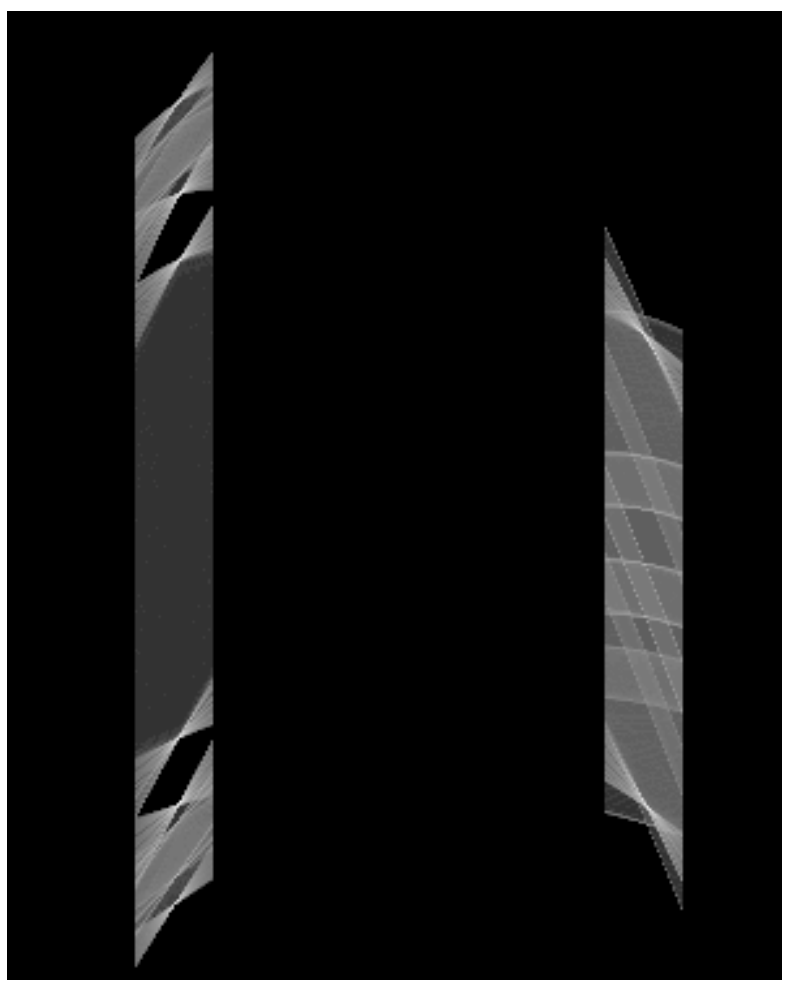

Fig. 10: The Hough transform of the processed image shows clear peaks 


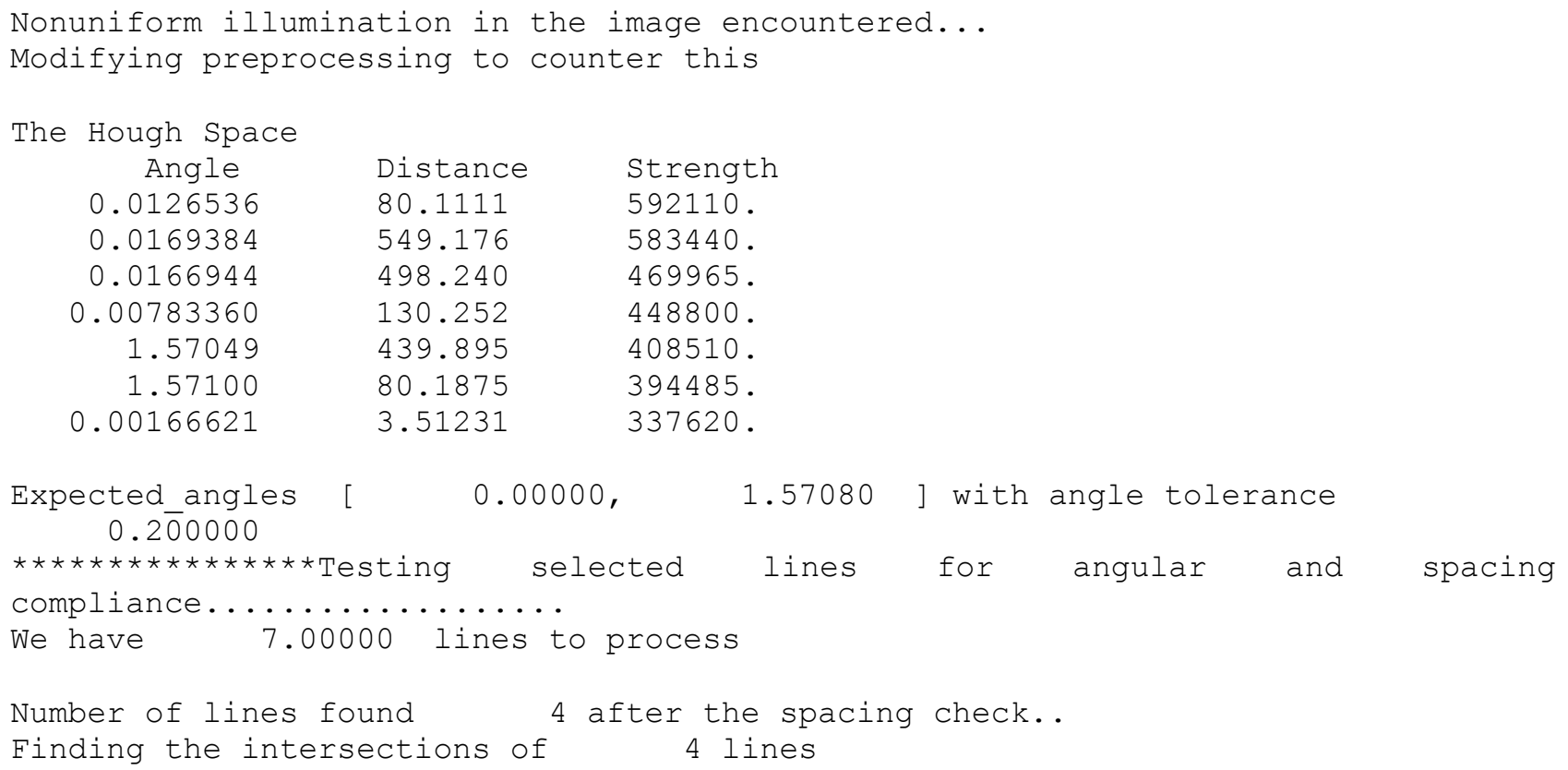

Fig. 11: The Hough transform results from the algorithm

Note from the output that the algorithm detects five near 0 degree lines which have the highest peak values. The two closest lines with the line spacing are selected after the spacing test. Similarly the two 90 degree lines are tested for proper spacing. Thereafter the intersection of the four lines are calculated

\section{TESTING WITH A ROTATED BEAM}

Next we test the algorithm on a simulated image. Compared to the real beam, this image has a slightly bigger inner beam width. The beam is rotated by -5.73 degree or -0.1 radians. A typical algorithm output shows the image variation to be negligible (sigma $=0.05$ ) in the $\mathrm{x}$-direction. Thus without additional pre-processing an edge detection followed by 


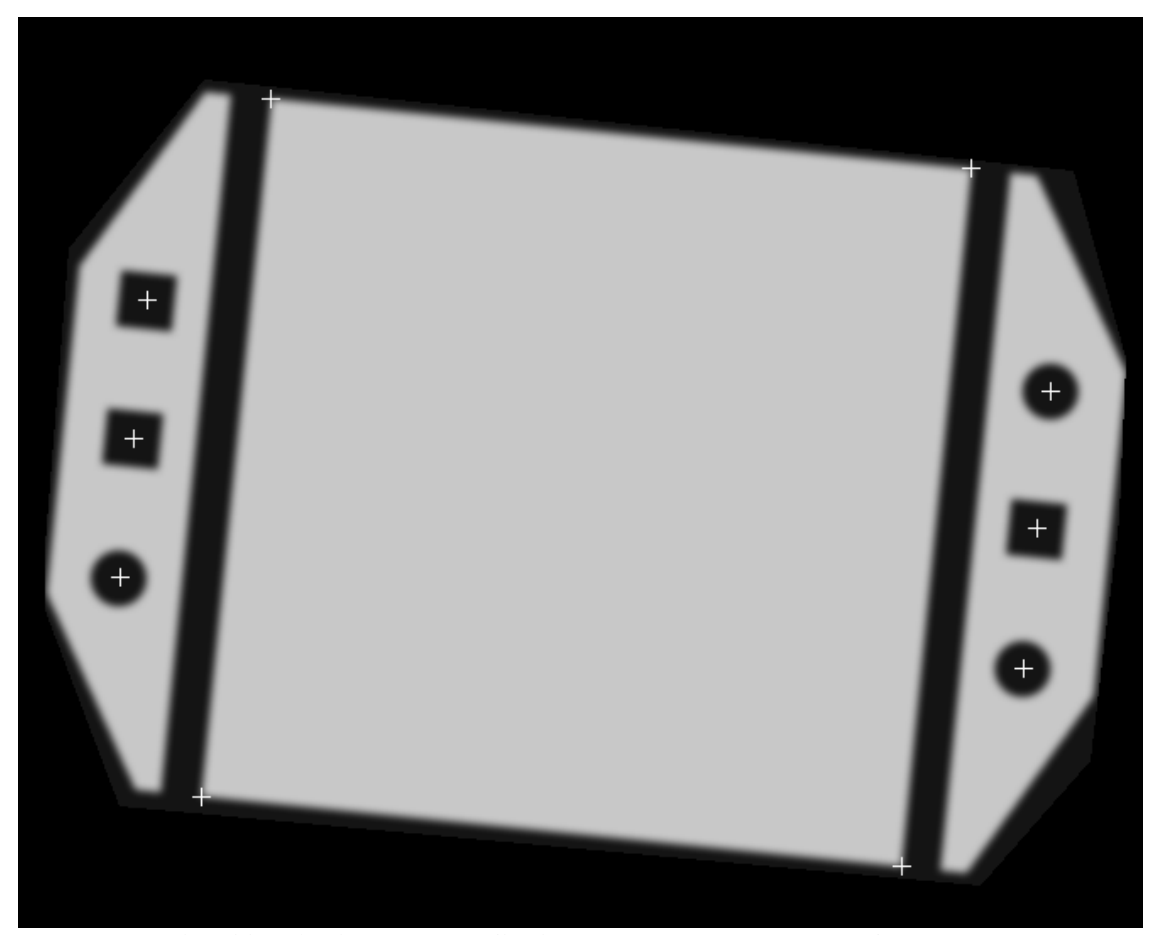

Fig. 12: A simulated ISP image rotated by -5.73 degree

a Hough transform is performed. As shown in the algorithm output Fig. 13, the peaks are detected at 1.47 radians instead of 1.57 radians. Also, instead of 0 radians, we see -0.1 radians. The change in the angle is also evident in the filtered

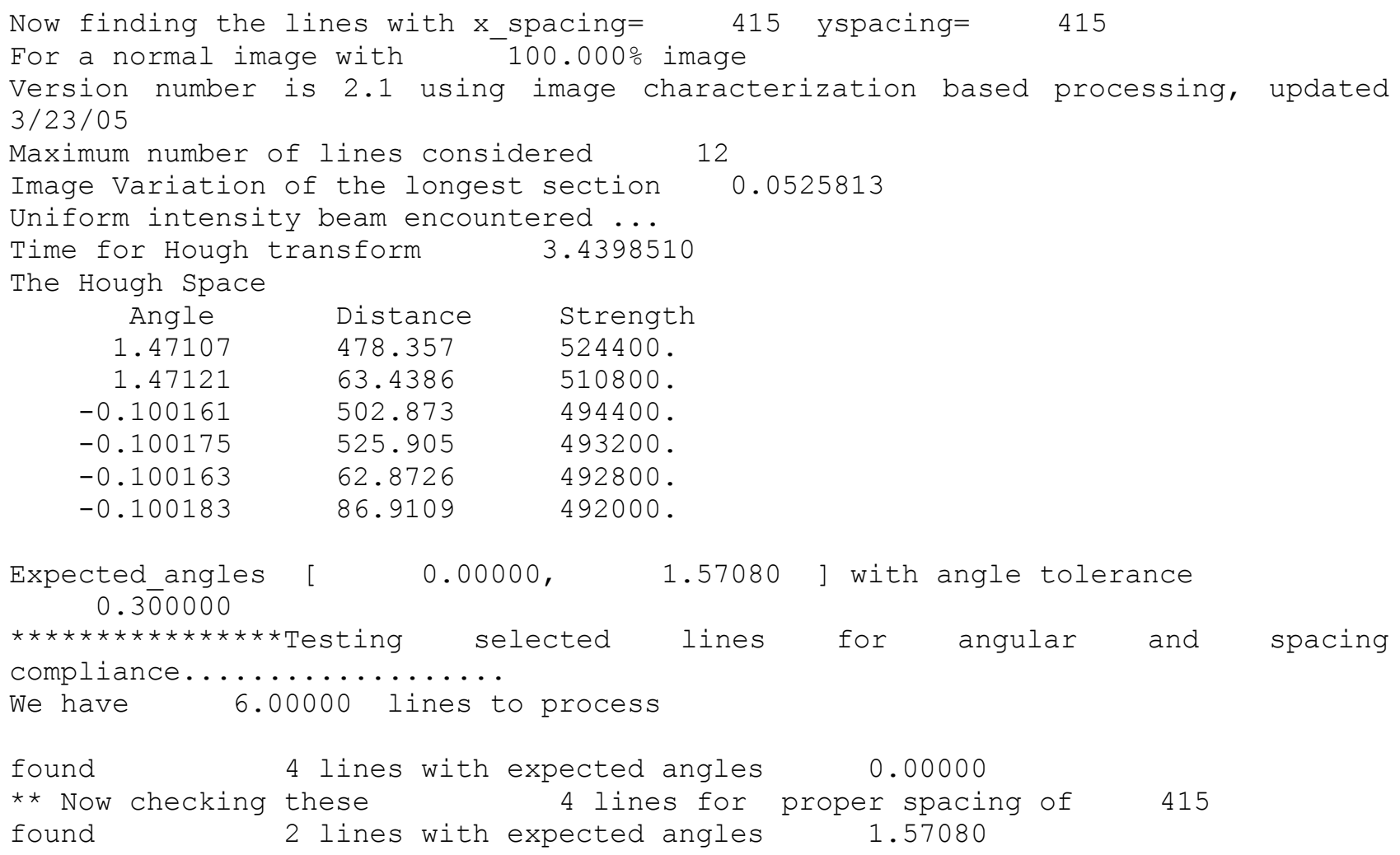


$\star \star$ Now checking these
Number of lines found

Finding the intersections of

92.8251

506.724

134.322

54.4794

13.1223

548.207
4 lines

0.239400

0.239400

0.239400

0.239400

Fig. 13: The output from the algorithm for rotated image in Fig. 12

Hough transform picture as shown in Fig. 14. Note that the peaks are shifted to the left of the center frequencies of 0 radians and 1.57 radians. One can compare Fig. 14 with Fig. 10, where the peaks are at the center of the pass band. Again spacing test is used to select the correct lines.

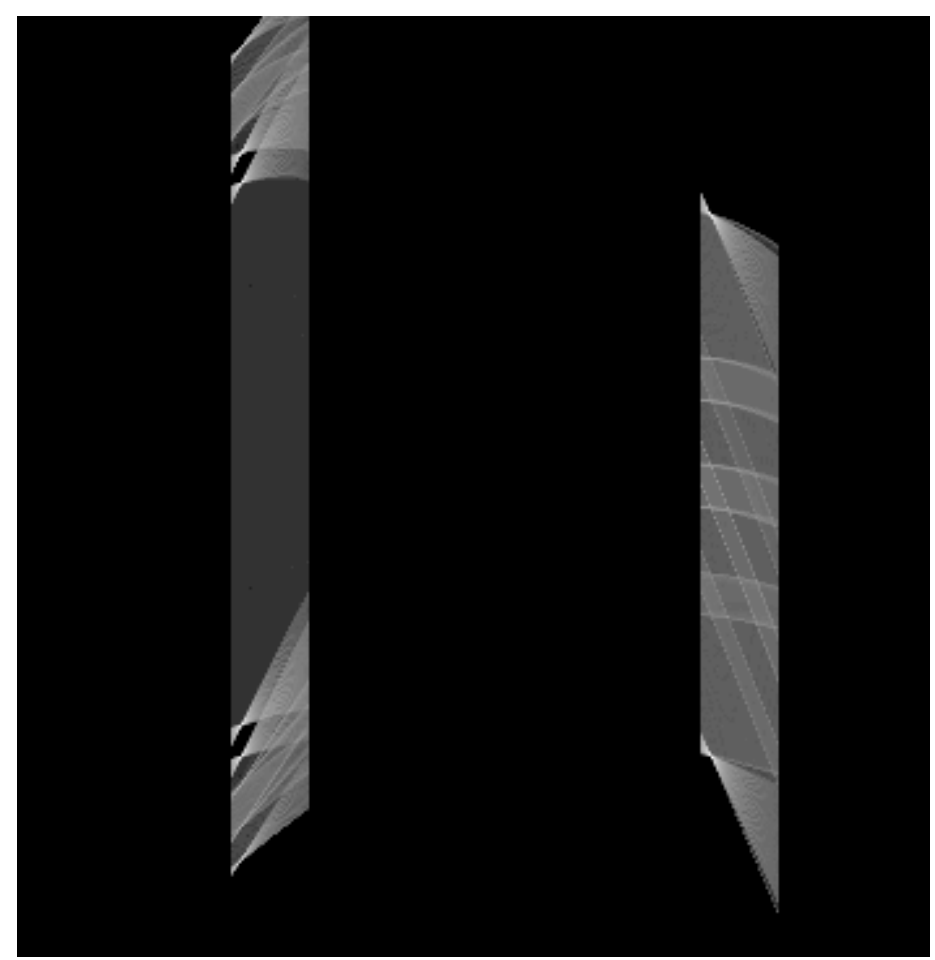

Fig. 14: The Hough transform of the processed image shows clear peaks

\section{CONCLUSIONS}

In this paper, a method of detection of beam rotation necessary for commissioning new beam lines is presented. The method is based on detecting corners of the square beam using Hough transform based processing. We show that in the presence of uniform illumination, we apply the spacing tolerance to filter out the proper lines of interest. In the presence of varying illumination, we apply additional processing to overcome the effect of nonuniform illumination the lines. The algorithm is made adaptive by incorporating the image quality detection and consequent processing for that type of image.

\section{ACKNOWLEDGEMENT}


This work was performed under the auspices of the U.S. Department of Energy by the University of California, Lawrence Livermore Laboratory under contract No. W-7405-Eng-48.

\section{REFERENCES}

1. E. Moses, et al., "The National Ignition Facility: Status and Plans for Laser Fusion and High-Energy-Density Experimental Studies", Fusion Science and Technology, V. 43, p. 420, May 2003.

2. P. V. C. Hough, "Methods and means for recognizing complex patterns," U. S. Patent 3,069,654.

3. R. O. Duda, and P. E. Hart, "Use of the Hough Transformation to Detect Lines and Curves in Pictures," Comm. ACM, Vol. 15, pp. 11-15, 1972.

4. M. A. Karim and A. A. S. Awwal, Optical Computing: An Introduction, John Wiley, New York, NY, 1992. 